\title{
Electron transport through monovalent atomic wires
}

\author{
Lee, Y. J.; Brandbyge, Mads; Puska, M. J.; Taylor, Jeremy Philip; Stokbro, Kurt; Nieminen, R. M.
}

Published in:

Physical Review B Condensed Matter

Link to article, DOI:

10.1103/PhysRevB.69.125409

Publication date:

2004

Document Version

Publisher's PDF, also known as Version of record

Link back to DTU Orbit

Citation $(A P A)$ :

Lee, Y. J., Brandbyge, M., Puska, M. J., Taylor, J. P., Stokbro, K., \& Nieminen, R. M. (2004). Electron transport through monovalent atomic wires. Physical Review B Condensed Matter, 69(12), 125409.

https://doi.org/10.1103/PhysRevB.69.125409

\section{General rights}

Copyright and moral rights for the publications made accessible in the public portal are retained by the authors and/or other copyright owners and it is a condition of accessing publications that users recognise and abide by the legal requirements associated with these rights.

- Users may download and print one copy of any publication from the public portal for the purpose of private study or research.

- You may not further distribute the material or use it for any profit-making activity or commercial gain

- You may freely distribute the URL identifying the publication in the public portal 


\title{
Electron transport through monovalent atomic wires
}

\author{
Y. J. Lee, ${ }^{1}$ M. Brandbyge, ${ }^{2}$ M. J. Puska,${ }^{1}$ J. Taylor, ${ }^{2}$ K. Stokbro, ${ }^{2}$ and R. M. Nieminen ${ }^{1}$ \\ ${ }^{1}$ COMP/Laboratory of Physics, Helsinki University of Technology, P.O. Box 1100, FIN-02015 HUT Helsinki, Finland \\ ${ }^{2}$ Mikroelecktronik Centret (MIC), Technical University of Denmark, Bldg 345E, DK-2800 Lyngby, Denmark
}

(Received 4 September 2003; revised manuscript received 13 November 2003; published 19 March 2004)

\begin{abstract}
Using a first-principles density-functional method we model electron transport through linear chains of monovalent atoms between two bulk electrodes. For noble-metal chains the transport resembles that for free electrons over a potential barrier whereas for alkali-metal chains resonance states at the chain determine the conductance. As a result, the conductance for noble-metal chains is close to one quantum of conductance, and it oscillates moderately so that an even number of chain atoms yields a higher value than an odd number. The conductance oscillations are large for alkali-metal chains and their phase is opposite to that of noble-metal chains.
\end{abstract}

DOI: 10.1103/PhysRevB.69.125409

PACS number(s): 73.63.Rt, 73.23.Ad

Chains of metal atoms can be considered as ultimate conductors of the future nanoelectronics. On the other hand, their experimental and theoretical investigation enlightens fundamental physical issues related to the confinement of electron states. ${ }^{1}$ Especially, an interesting question is how the valency $^{2}$ and the different responses of different types of atomic states ( $s, p$, and $d$ orbitals) are reflected in the various properties of the atomistic constrictions. A possible realization of conducting atom chains is a string of gold atoms adsorbed on a silicon surface. ${ }^{3}$ Moreover, the formation of free-standing chains of gold atoms between two bulk electrodes was predicted by molecular-dynamics calculations 4 and verified by high-resolution transmission electron microscopy ${ }^{5}$ and mechanically controlled break junction (MCBJ) experiments. ${ }^{6}$

Transport properties of atomic contacts have been investigated for more than a decade. ${ }^{1}$ The well established quantization of conductance of gold contacts and chains has made them benchmark systems. In the MCBJ experiments it is possible to measure conductance and chain-length distributions simultaneously and thereby correlate these measures. ${ }^{7}$ Very recently, it has been reported that the conductance of chains of $\mathrm{Au}, \mathrm{Pt}$, and Ir atoms oscillates as a function of the number of atoms in the chain. ${ }^{8}$ However, the detailed structure of chain, including the actual number of chain atoms, remains unknown in the MCBJ measurements. This clearly calls for support from first-principles calculations.

In the present work we consider chains of monovalent alkali-metal (AM) and noble-metal (NM) atoms. When pulling them in experiments, the conductance is just before breaking close to one quantum ( $\left.1 G_{0}=2 e^{2} / h\right)$ signaling that only one channel is open for transmission. ${ }^{1}$ Theoretically, the conductance of $\mathrm{Na}$ atom chains has been studied with density-functional electronic-structure calculations. ${ }^{9-11}$ Sim et $a l .{ }^{10}$ predicted using the Friedel sum rule that the conductance of a chain of an odd number of $\mathrm{Na}$ atoms between bulk electrodes is close to $1 G_{0}$ whereas that for a chain of an even number of atoms is clearly below $1 G_{0}$. This was confirmed by Tsukamoto and Hirose. ${ }^{11}$ The findings for the $\mathrm{Na}$ atom chains can be rationalized ${ }^{10}$ by noting that the atom chain exhibits strong molecular-orbital-like resonances, the positions of which relative to the Fermi level are determined by the charge neutrality requirement. Recently, Thygesen and Jacobsen $^{12}$ calculated conductances of $\mathrm{Al}$ atom chains. The conductance shows oscillations with a period of four Al atoms. The results can be understood by the resonance formation, but instead of the charge neutrality requirement the electronic structure of an infinite wire has to be used in the interpretation.

Several works have addressed also the conductance of gold wires on the basis of electronic-structure calculations. ${ }^{13-15}$ These works show that the chains have a tendency to dimerize upon strong elongation in accord with the Peierls mechanism. However, the conductance as a function of the chain length has not been studied consistently. Brandbyge et al. ${ }^{16}$ calculated the transmission for gold chains of two different lengths using the tight-binding formalism with the local charge neutrality requirement. The chain with an even number of atoms showed a slightly larger conductance than that with an odd number of atoms.

We calculate the conductance versus the chain length for AM and NM chains connected to electrodes. Although both types of systems can be considered as monovalent we find unexpected differences in the electron transport near the Fermi level. Their origin is in the hybridization of the $d$ orbitals with the $s$ orbitals in the NM systems. Surprisingly, the transport through the NM chains is free-electron-like whereas that for AM chains is dominated by strong resonances of molecular-orbital character.

We perform electronic-structure calculations within the density-functional theory and the local-density approximation for electron exchange and correlation. Nonlocal normconserving scalar-relativistic pseudopotentials with partial core-corrections and atomic-orbitals basis sets of the SIESTA code are used. ${ }^{17,18}$ The electronic structures and transport properties are determined using the nonequilibrium Green'sfunction method based on the TRANSIESTA package. ${ }^{19}$

The geometry used for the nanoconstrictions is shown in Fig. 1(a). The leads have the face-centered-cubic (fcc) structures and the atom chain is in the [100] direction. The calculation volume (repeated periodically perpendicular to the wire) is shown in the figure. The chain atoms (with the coordination number of 2) are labeled by successive integers. The tip atoms labeled by $T_{\mathrm{L}}$ and $T_{\mathrm{R}}$ belong to the electrodes. 

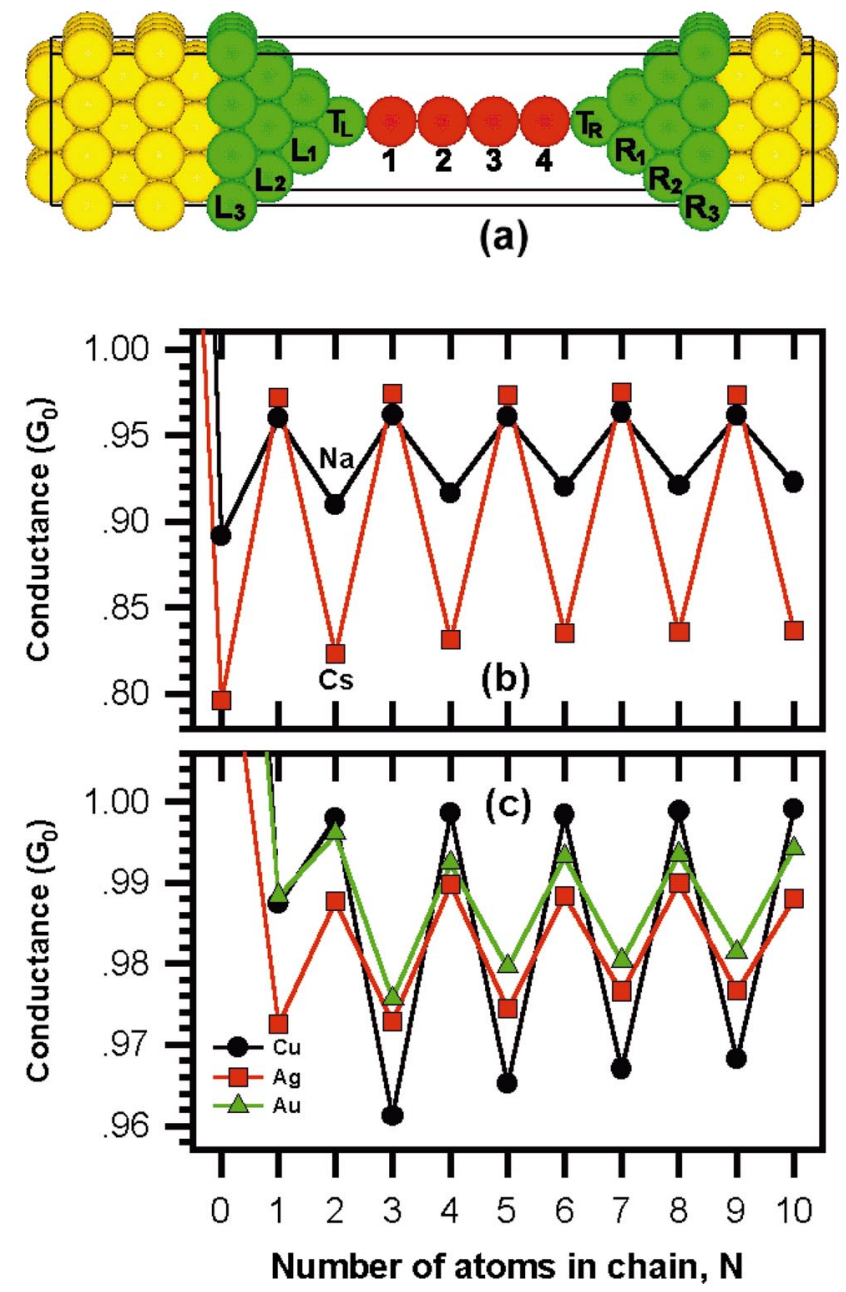

FIG. 1. (Color) (a) Model used for nanoconstrictions. The chain and cone atoms are denoted by red and green circles, respectively. For these atoms the electronic structure is solved self-consistently. For the other atoms in the calculation supercell, denoted by yellow circles, the effective potential of the bulk atoms is used. The borders of the supercell are indicated by thin solid lines. (b) Conductance of $\mathrm{Na}$ (black circles) and $\mathrm{Cs}$ (red squares) chains and (c) that of $\mathrm{Cu}$, $\mathrm{Ag}$, and $\mathrm{Au}$ chains as a function of the number of atom in the chain.

The successive atomic layers in the left and right leads are labeled as $L_{i}$ and $R_{i}, i=1, \ldots, 3$, respectively. In the calculation volume there are totally $109+N$ atoms, with $N$ atoms in the chain. The electronic structure corresponding to 46 $+N$ atoms is solved self-consistently, and for the remaining atoms the effective potential is frozen to the value calculated self-consistently for the bulk system. We have tested that the enlargement of the supercell does not change significantly the results obtained. We will focus on the effects of the electronic structure on the conductance. Therefore we use the same structure, with the experimental bulk nearest-neighbor atom distances $\left(r_{0}\right)$ in the chain, for both the AM and NM systems. The distances $r$ 。 used are $2.630 \AA(\mathrm{Na}), 3.762 \AA$ (Cs), $2.556 \AA(\mathrm{Cu}), 2.889 \AA(\mathrm{Ag})$, and $2.886 \AA(\mathrm{Au})$.

Figures 1(b) and 1(c) show the conductances of the AM ( $\mathrm{Na}$ and $\mathrm{Cs})$ and $\mathrm{NM}(\mathrm{Cu}, \mathrm{Ag}, \mathrm{Au})$ chains, respectively. For the AM chains the conductance is close to $1 G_{0}$ for odd $N$ and for even $N$ the conductance is clearly below $1 G_{0}$. These

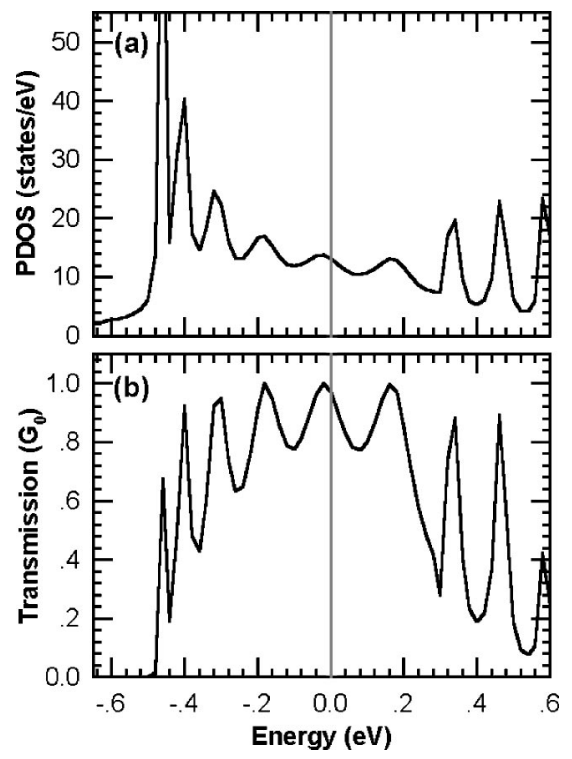

FIG. 2. Cs nanoconstriction with $N=9$ chain atoms. (a) Density of states projected (PDOS) onto the chain and the tip atoms. (b) Total transmission. The energy is given relative to the Fermi level $\left(E_{F}=0\right)$.

even-odd oscillations have been reported in theoretical studies for Na wires ${ }^{9-11}$ for various types of electrode models. Our finding that similar oscillations are found also when using fcc electrodes means that the result does not depend strongly on the detailed structure of the electrode. The origin of the conductance oscillations is an induced resonance state which is well localized at the chain. ${ }^{10,20}$ Due to the charge neutrality requirement the resonance is half filled for an odd $N$ causing a high local density of states (LDOS) at the Fermi level $\left(E_{F}\right)$ in the chain. Therefore the conductance has a maximum. For even $N$ the resonance is filled and well below $E_{F}$ resulting in a low LDOS at $E_{F}$ and a low conductance. The behavior of the conductance of the NM chains differs from that of the AM chains. The phase of the oscillations is opposite to that for the AM chains and the minima drop only a few percent below $1 G_{0}$.

Also the density of states (DOS) and the electron transmission show important differences between the AM and NM systems. Figures 2 and 3 show the projected density of states (PDOS) and the energy-dependent transmission coefficient for the Cs chain with $N=9$ and for the Au chain with $N=10$, respectively. The PDOS includes the chain and the two tip atoms. The overall PDOS shape of the Cs chain decays toward high energies as is typical for a onedimensional system. However, there are strong peaks superimposed. They correspond to resonance states arising from the chain molecular orbitals with zero, one, two, etc., nodes along the chain. For a chain of $N$ atoms there are $N / 2$ resonance peaks below $E_{F}$. Thus, our notation that only the atoms with the coordination number of 2 belong to the chain attains a real physical meaning. The corresponding resonance peaks dominate also the transmission as can be seen in Fig. 2(b). Thus, our results confirm the model by Sim et al.

Figure 3 shows the PDOS and transmission as a function of energy for the Au nanoconstriction with $N=10$. Since 

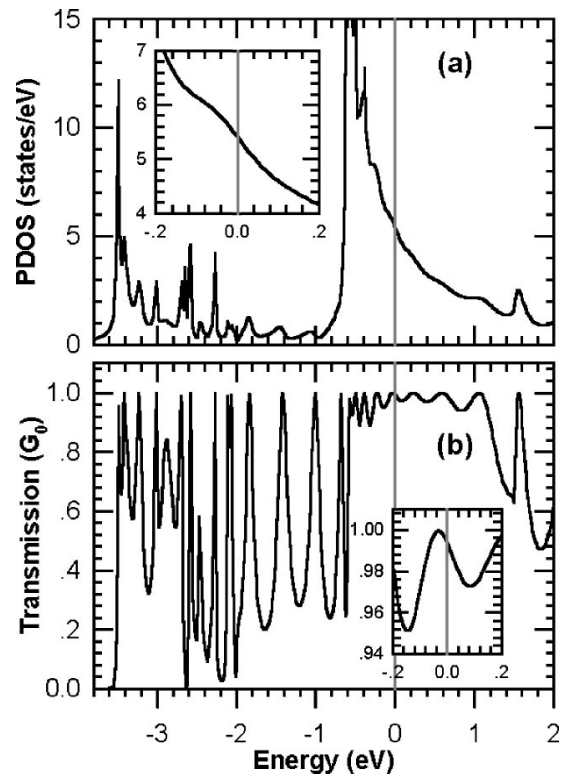

FIG. 3. Au nanostriction with $N=10$ chain atoms. (a) Density of states projected on the $s$ orbitals ( $s$-PDOS) of the chain and the tip atoms. (b) Transmission of the $s$ channel. The energy is given relative to the Fermi level $\left(E_{F}=0\right)$. The insets show magnifications of PDOS and transmission near the Fermi level.

only a single eigenchannel is open near $E_{F}$, the corresponding $s$-projected PDOS and $s$-channel transmission are shown in Fig. 3. Below $-0.6 \mathrm{eV}$ the $s$-PDOS shows strong oscillations, resembling the oscillations in the total-PDOS for the $\mathrm{Cs}$ chain in Fig. 2(b). For energies above $-0.6 \mathrm{eV}$ the $s$-PDOS decays as the DOS in a one-dimensional system. Resonance states now appear as weak oscillations giving rise to shoulders in the PDOS. For the ten atom chain $E_{F}$ is located between two shoulders of the curve, while for a chain with an odd $N E_{F}$ is located at a shoulder. In the same energy region the transmission behaves free-electron-like, i.e., with transmission coefficients close to unity. However, there are small oscillations in the transmission coefficients, and interestingly the resonance shoulders now correspond to transmission minima. As we will illustrate with a simple model below, this behavior is related to interference of the incident wave with the wave reflected twice at the chain ends. In Fig. 3(b) for $N=10 E_{F}$ is close to a transmission maximum. This is true also for other even $N$ values, while for odd $N E_{F}$ lies close to transmission minima. According to our results this behavior is valid for other noble metals, too. This interplay between the Fermi level and the transmission results in the conductance with the even-odd oscillations in Fig. 1(c).

Next we want to understand from the basic electronic structures why the transport is free-electron-like in the NM chains whereas in AM chains it is dominated by the resonances due to the molecular orbitals. The excess Mulliken charges per atom projected on the different atoms of the Cs and Au systems with $N=10$ are shown in Fig. 4(a). For Au there is strong charge transfer so that the tip and the chain end atoms gain extra electron charge. The strong charge transfer can be understood as follows. At low-coordinated $\mathrm{NM}$ atoms, the electrons in the uppermost antibonding $d$

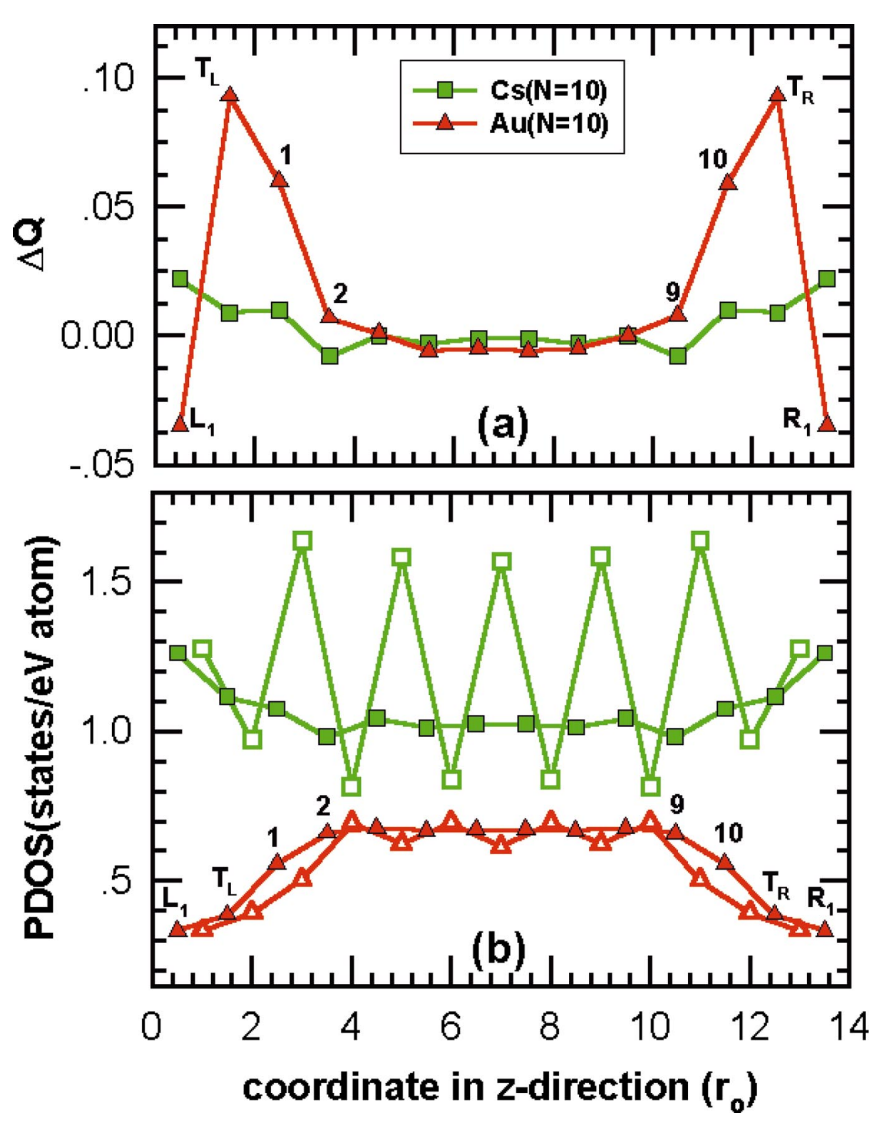

FIG. 4. (Color) (a) Excess Mulliken charge for different atoms in the $\mathrm{Cs}$ and $\mathrm{Au}$ nanoconstrictions with $N=10$ chain atoms. For the labeling of the atoms, see Fig. 1(a). (b) Density of states at $E_{F}$ projected on the different atoms in the $\mathrm{Cs}$ (squares) and $\mathrm{Au}$ (triangles) nanoconstrictions with $N=9$ (open markers) and $N=10$ (filled markers) chain atoms.

states which are in the region of ion cores are transferred to the nonbonding $s$ states which are away from ions (vacuum region) in comparison with the bulk atoms. ${ }^{21}$ The driving forces of this rehybridization are both the increase of the bonding by reducing the electrons in the antibonding $d$ states and the reduction of the kinetic energy by relaxing the electrons from ion cores toward vacuum. ${ }^{21}$ Also the electrons in bonding $s$ states are slightly relaxed toward vacuum to reduce the kinetic energy. The spill-out of the electrons toward vacuum by the rehybridization and the pure relaxation mechanisms increases the work function of the lowcoordinated NM systems. When the chain is connected to the leads the work function difference causes charge transfer from the leads to the chain because the spill-out is larger for the chain atoms than for the surface atoms. A dipole is formed so that the potential in the chain rises relative to that in the leads. The $d$ bands are shifted upwards relative to $E_{F}$ when comparing the LDOS in the chain to that in the leads. This phenomenon is observed for Au chains also in the tightbinding calculations with the local charge neutrality. ${ }^{16}$ For the AM systems with no electron in antibonding states the spill-out of the electrons in bonding states toward vacuum should lead to the weakening of bonds. Therefore both the spill-out and concurrently the charge transfer would be weak. 
Indeed in the case of AM the week charge transfer is observed as shown in Fig. 4(a). Next we argue that the strong charge transfer in the NM systems causes the transport properties to differ from the AM chains.

In the AM systems electron scattering takes place mainly at the connections between the chain and the leads. As shown in Fig. 2 resonances corresponding to molecular orbitals are formed and they are filled according to the charge neutrality requirement for the atom chain. ${ }^{10,20}$ This picture is confirmed in the PDOS for the $N=9$ and $N=10$ Cs nanoconstrictions in Fig. 4(b). Here the projection is performed at $E_{F}$ on different individual atoms. The even-odd oscillations in the PDOS for the odd $N$ correspond to a molecular state which vanishes at every second atom. For even $N$ the uppermost occupied molecular orbital has a similar amplitude on every chain atom, and there are no clear oscillations.

This scattering at the connection points is also present in the NM chains. If this were the only scattering mechanism we would obtain maximum transmission for odd $N$ also for $\mathrm{NM} .{ }^{20}$ However, for the NM systems, this scattering is weak due to the stronger coupling (higher DOS) to the electrodes. This is reflected in the high transmission for both odd and even $N$ for NM. The weak chain-electrode scattering makes additional scattering mechanisms inside the NM chains visible.

The PDOS for the Au chain in Fig. 4(b) does not show strong oscillations in accord with the absence of the molecular-orbital character. The PDOS values rise from the leads to the chain corresponding to the potential barrier inside the chain caused by the charge-transfer dipoles. For the NM nanoconstrictions this barrier dominates the scattering near $E_{F}$ while the scattering at the connection points gives rise to the molecular-orbital-like resonances well below $E_{F}$ [see Fig. 3 (a)]. The electron transport is free-electron-like near $E_{F}$ and the transmission in Fig. 3(b) obeys the equation for free electrons over a one-dimensional potential barrier, i.e.,

$$
T(E)=\left[1+\frac{V_{0}^{2} \sin ^{2}(k L)}{4 E\left(E-V_{0}\right)}\right]^{-1},
$$

where $V_{0}$ and $L$ are the barrier height and length, respectively. $k=\sqrt{2\left(E-V_{0}\right)}$ is the wave vector inside the barrier region. According to Eq. (1) the transmission minima (maxima) appear when $k L=n \pi / 2$ with $n$ equal to an odd (even) integer. Now we need a relation between the Fermi wave vector $k_{F}$ and $n$. For an infinite chain of monovalent atoms $k_{F}=\pi /(2 a)$, where $a$ is the interatomic distance. For a finite wire with $N$ atoms this translates into $k_{F}=n \pi / 2 L$, with $L=N a$. To justify this relation we have calculated $k_{F}$ of the barrier model numerically, assuming that the barrier region is charge neutral. Indeed we find $k_{F} \approx n \pi / 2 L$ when $n$ is even. Thus this simple barrier model explains the behavior of the transmission through the NM chains.

Conductances of $\mathrm{Au}$ atom chains have been measured recently by Smit $e t a l{ }^{8}$ using the MCBJ method. The conductance as a function of the Au atom chain length in individual pulls shows steps which are of the order of $0.1 G_{0}$, i.e., remarkably larger than the oscillations in our results for the

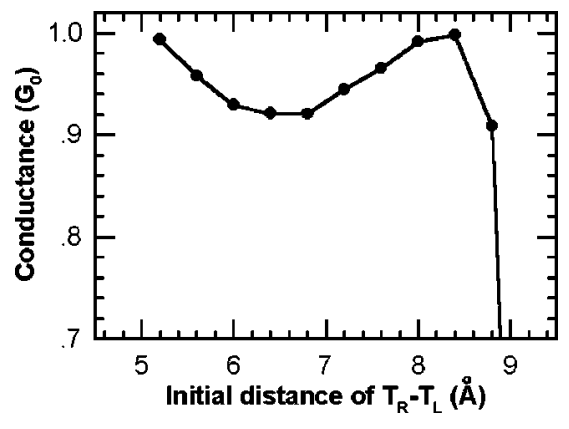

FIG. 5. Conductance of the $\mathrm{Au}$ nanoconstriction with $N=2$ chain atoms as a function of the stretching. The stretching is given as the initial distance between the tip atoms $T_{\mathrm{R}}$ and $T_{\mathrm{L}}$ [see Fig. 1 (a)] before relaxing the atomic positions.

Au chains with constant interatomic distances. Similar steps were recorded earlier by Rubio-Bollinger et $a l^{22}$ Furthermore, Smit et al. concluded that the maxima (minima) in the conductance correspond to an odd (even) number of atoms in the chain. This is also in contrast to our results in Fig. 1(c). The findings by Smit et al. resemble the conductance oscillations of the AM chains in Fig. 1(b). This behavior arises from the changes in the electronic structure as a function of the number of chain atoms. However, according to our calculations the electronic structures of the NM chains behave differently, and it is not justified to apply the models developed for the AM chains to interpret the NM chain results.

In addition to the purely electronic effects discussed above, the changes of atomic structure when a chain is pulled may affect the conductance behavior. In order to estimate the magnitude of these effects, we have calculated the electronic and the relaxed atomic structures along the conductance for the Au constriction with $N=2$ chain atoms as a function of the elongation of the system. The starting point is the same configuration as discussed above but now with a different distance between the tip atoms $T_{\mathrm{R}}$ and $T_{\mathrm{L}}$ set up by changing the (equal) bond lengths between the "dimer" chain and tip atoms. Then we allow all the atoms except the buffer layer atoms [yellow circles in Fig. 1(a)] to relax in order to find their equilibrium positions. The calculation is repeated for a new longer initial distance between the tip atoms $T_{\mathrm{R}}$ and $T_{\mathrm{L}}$ until the wire breaks in the simulation with an abrupt decrease in the conductance. The conductance is shown in Fig. 5 as a function of the elongation. The conductance varies between $1.00 G_{0}$ and $0.92 G_{0}$. This variation is correlated with changes in interatomic distances in the atom chain. The electronic structure remains at all elongation stages freeelectron-like, i.e., there are no strong resonances in the DOS near the Fermi level. The conductance variation in Fig. 5 is much larger than the oscillations seen in Fig. 1(b). Thus, the effect of the stretching on the conductance is larger than that of adding a new atom in a chain when keeping the interatomic distances constant.

To model the experimental situation in detail we would have to involve many more atoms in order to simulate how atoms are pulled from the bulk banks of the electrodes into the chain. Moreover a number of different initial geometries would probably have to be performed. This is well beyond 
the limitations of first-principles simulation of today. However, it is interesting to note that according to our preliminary results the conductance as a function of elongation behaves differently for different $N$. For example, for $N=3$ the conductance variation is smaller than for $N=2$. The stretching effects might explain the experimental finding that the conductances of the Pt and Ir atom chains oscillate similarly to that of the Au chains but with larger amplitude. ${ }^{8}$ Compared to $\mathrm{Au}$ the conductance of $\mathrm{Pt}$ chains is expected to depend more strongly on the change in bond distance due to the conduction channels involving the $d$ electrons. ${ }^{23}$

In conclusion, we have studied electron-transport properties of AM and NM atom chains from first principles. In the NM nanoconstrictions the lowering of the atomic coordination results in charge transfer from uppermost $d$ orbitals to $s$ orbitals. A potential barrier is built up at the atom chain and the electron transport over it resembles that of free electrons. The conductance of the NM chains is close to one quantum of conductance and it oscillates as a function of the chain length so that conductance maxima (minima) occur at an even (odd) number of atoms in the chain. This is in contrast to the transport through AM chains for which the resonances due to the molecular states are dominating and result in strong even-odd conductance oscillations with the phase opposite to that of NM chains. Our simulations for the Au atom chains indicate that the conductance changes remarkably during the stretching of the wire. Our notions are important when interpreting the recent MCBJ results for $\mathrm{Au}$ chains. The model valid for AM atom chains cannot be used for NM atom chains. Moreover, on the ultimate limit of the atomic chain noble-metal atomic wires with a rather structureless free-electron-like conductance would be superior over the alkali-metal atomic wires showing strong resonance effects.

This work was supported by Academy of Finland through the Center of Excellence Program (2000-2005), and the Danish Natural Research Council (SNF). We acknowledge the generous computing resources of the Center for the Scientific Computing (CSC), Espoo, Finland.
${ }^{1}$ For a recent review see N. Agrait, A.L. Yeyati, and J.M. van Ruitenbeek, Phys. Rep. 77, 81 (2003).

${ }^{2}$ E. Scheer et al., Nature (London) 394, 154 (1998).

${ }^{3}$ P. Segovia, D. Purdie, M. Hengsberger, and Y. Baer, Nature (London) 402, 504 (1999).

${ }^{4}$ M.R. Sørensen, M. Brandbyge, and K.W. Jacobsen, Phys. Rev. B 57, 3283 (1998).

${ }^{5}$ H. Ohnishi, Y. Kondo, and K. Takayanagi, Nature (London) 395, 780 (1998).

${ }^{6}$ A.I. Yanson et al., Nature (London) 395, 783 (1998).

${ }^{7}$ C. Untiedt et al., Phys. Rev. B 66, 085418 (2002).

${ }^{8}$ R.H.M. Smit, C. Untiedt, G. Rubio-Bollinger, R.C. Segers, and J.M. Ruitenbeek, Phys. Rev. Lett. 91, 076805 (2003).

${ }^{9}$ N.D. Lang, Phys. Rev. Lett. 79, 1357 (1997).

${ }^{10}$ H.S. Sim, H.W. Lee, and K.J. Chang, Phys. Rev. Lett. 87, 096803 (2001).

${ }^{11}$ S. Tsukamoto and K. Hirose, Phys. Rev. B 66, 161402 (2002).

${ }^{12}$ K.S. Thygesen and K.W. Jacobsen, Phys. Rev. Lett. 91, 146801 (2003).

${ }^{13}$ M. Okamoto and K. Takayanagi, Phys. Rev. B 60, 7808 (1999).

${ }^{14}$ H. Häkkinen, R.N. Barnett, and U. Landman, J. Phys. Chem. B 103, 8814 (1999).

${ }^{15}$ T. Ono, H. Yamasaki, Y. Egami, and K. Hirose, cond-mat/0212603 (unpublished).
${ }^{16}$ M. Brandbyge, N. Kobayashi, and M. Tsukada, Phys. Rev. B 60, 17064 (1999).

${ }^{17}$ D. Sanchez-Portal, P. Ordejón, E. Artacho, and J.M. Soler, Int. J. Quantum Chem. 65, 453 (1999); J.M. Soler et al., J. Phys.: Condens. Matter 14, 2745 (2002).

${ }^{18}$ Basis-set: For NM, we used double- $\zeta$ (for $s$ and $d$ ) with singlepolarization (for $p$ ) sets. For AM, we used double- $\zeta$ (for $s$ ) with double polarization (for $p$ ) sets plus single- $\zeta$ functions for the unoccupied $d$ orbitals. All basis sets were optimized by minimizing the total energy with the constraint that the orbital range $\left(r_{c}\right)$ is smaller than 1.3 times the experimental bulk bond length. Our results for the odd-even transmission oscillations were also reproduced using the less accurate single- $\zeta+$ polarization basis sets.

${ }^{19}$ M. Brandbyge, J.-L. Mozos, P. Ordejon, J. Taylor, and K. Stokbro, Phys. Rev. B 65, 165401 (2002).

${ }^{20}$ F. Yamaguchi, T. Yamada, and Y. Yamamoto, Solid State Commun. 102, 779 (1997).

${ }^{21}$ Y. J. Lee et al. (unpublished).

${ }^{22}$ G. Rubio-Bollinger, S.R. Bahn, N. Agraï, K.W. Jacobsen, and S. Vieira, Phys. Rev. Lett. 87, 026101 (2001).

${ }^{23}$ S.K. Nielsen, Y. Noat, M. Brandbyge, R.H.M. Smit, K. Hansen, L.Y. Chen, A. I Yanson, F. Besenbacher, and J.M. van Ruitenbeek, Phys. Rev. B 67, 245411 (2003). 